\title{
Sorafenib suppresses hepatitis B virus gene expression via inhibiting JNK pathway
}

\author{
Hyun Kook Cho', Ju Ran Kim¹, So Young Kim¹, Yi Yi Kyaw', Aye Aye Win², \\ Jae Hun Cheong ${ }^{1}$
}

${ }^{1}$ Department of Molecular Biology, Pusan National University, Busan 609-735, South Korea

${ }^{2}$ Department of Medical Research (Lower Myanmar), Experimental Medicine Research Division, Yangon 11191, Myanmar

\begin{abstract}
Aim: Hepatitis B virus (HBV) infection is a major cause of chronic liver diseases. Sorafenib is a multikinase inhibitor and an approved anti-liver cancer drug. Here we demonstrated the antiviral effect of sorafenib on HBV gene expression. Methods: To investigate the effect of sorafenib on HBV gene expression, a luciferase assay was performed with $\times 1.3$ Cp-luciferase HBV construct and reverse transcriptase polymerase chain reaction (PCR), real-time PCR, and Western blotting analyses were performed using HepG2 cells derived from hepatocellular carcinoma and Chang liver cells derived from a normal liver tissue. Results: Sorafenib suppressed HBV gene expression via inhibiting the JNK pathway. In this process, the farnesoid $X$ receptor ( $F X R)$, a transcription factor that has been reported to increase HBV replication and gene expression, was under control of the JNK pathway. Notably, JNK activation increased FXR protein levels, not mRNA levels. Conclusion: Sorafenib suppressed HBV gene expression via inhibiting the JNK pathway, which regulates FXR activity.
\end{abstract}

Key words: Sorafenib; hepatitis B virus; JNK; farnesoid X receptor; antiviral effect

Address for correspondence:

Prof. Jae Hun Cheong, Department of Molecular Biology, Pusan National University, Busan 609-735, South Korea.

E-mail: molecule85@pusan.ac.kr

Received: 04-02-2015, Accepted: 27-04-2015

\section{INTRODUCTION}

Hepatitis B virus (HBV) infection is a serious public health problem with approximately 350 million people with chronic HBV infection in the world. Among HBV-infected patients, $15-40 \%$ develop cirrhosis, liver failure, and hepatocellular carcinoma (HCC). ${ }^{[1]}$ Persistent infection with HBV is a leading cause of human chronic liver disease. It is well known that for cancer patients with chronic HBV infection who are undergoing cytotoxic chemotherapy, hepatic dysfunction occurs more frequently than that of non-HBV carriers, and this has been attributed mainly to the development of

\begin{tabular}{|l|c|}
\hline \multicolumn{2}{|c|}{ Access this article online } \\
\hline \multirow{2}{*}{ Website: } & Quick Response Code \\
\hline http://www.hrjournal.net/ & \\
DOI: & \\
10.4103/2394-5079.158391 & \\
\end{tabular}

HBV reactivation. HBV reactivation can be transient and resolve spontaneously but often leads to clinically apparent acute hepatitis. Its occurrence constantly results in delays in chemotherapy schedules and disruption of cytotoxic treatment regimens and in the most severe cases, leads to acute liver failure and death. ${ }^{[2]}$ Although HBV reactivation can be prevented by antiviral prophylaxis, the mechanism by which HBV contributes to events leading to liver injury in chronic HBV carriers who are receiving cancer chemotherapy remains to be fully understood.

Sorafenib (BAY43-9000, Nexavar) is the first and only medication approved by the USA Food and Drug Administration for the treatment of advanced HCC. Sorafenib is a multikinase inhibitor which has been shown to block tumor cell proliferation and angiogenesis by inhibiting serine/threonine kinases (c-RAF and b-RAF) as well as several receptor tyrosine kinases. ${ }^{[3]}$ Although several drugs with high efficacy against chronic hepatitis $\mathrm{B}(\mathrm{CHB})$ are currently approved in the USA for the treatment of $\mathrm{CHB}$, the drugs have adverse effects 
including: drug resistance, nephrotoxicity, and myopathy. ${ }^{[4]}$ Therefore, new drug strategies with other mechanisms are extensively required for antiviral therapy.

This study focused on the anti-viral effect of sorafenib based on the function of inhibiting the molecular signaling pathway. We investigated the effect of sorafenib on HBV replication using a human hepatoma cell line and a normal liver cell line and confirmed that sorafenib suppressed HBV replication via inhibiting the JNK pathway, which regulated the activity of the transcription factor, farnesoid $\mathrm{X}$ receptor (FXR). These results suggest that $\mathrm{HBV}$ replication is associated with the JNK pathway and may be regulated through inhibition of the JNK pathway by sorafenib.

\section{METHODS}

\section{Cell culture}

HepG2 and Chang liver cells (all obtained from the American Type Culture Collection, Manassas, VA, USA) were maintained in Dulbecco's Modified Eagle Medium with $10 \%$ fetal bovine serum (Gibco BRL, USA) and $1 \%(\mathrm{v} / \mathrm{v})$ penicillin-streptomycin (Gibco BRL, USA) at $37^{\circ} \mathrm{C}$ in a humid atmosphere containing $5 \% \mathrm{CO}_{2}$.

\section{Plasmid constructs and reagents}

The $\times 1.3$ Cp-luciferase HBV was generously provided by $Y$. Shaul (Weizmann Institute of Science, Rehovot, Israel). The 1.2 mer HBV including N-terminal $\times 3$ flagged HBx were kindly provided by W. S. Ryu (Yonsei University, Seoul, South Korea). To construct HBV-Xp-luc and HBV-preS1 p-luc, the promoter fragments in HBV genome construct were amplified by polymerase chain reaction (PCR) using cloning primers containing restriction enzyme site, HindIII, and KpnI. After digestion, the fragments were cloned into pGL4 vectors. The sequences were confirmed by automated DNA sequencing. Sorafenib was purchased from Selleckchem (Houston, TX, USA). Chenodeoxycholic acid (CDCA) and Z-guggulsterone (Z-GGS) were purchased from Sigma (St. Louis, MO, USA). SP600125 was purchased from Calbiochem (Billerica, MA, USA). The transfection reagents PolyFect and jetPEI were purchased from Qiagen (Hilden, Germany).

\section{Drug and inhibitor treatment}

Cells were treated with indicated chemicals or vehicle controls and incubated for $24 \mathrm{~h}$. Control vehicle treatment (dimethelsulfooxide) was equivalent to the dose range experiments for each tested drug.

\section{Luciferase assay}

Cells were transfected with both the reporter vector and the $\beta$-galactosidase expression plasmid along with each indicated expression plasmid using PolyFect. After transfection, the cells were lysed in cell culture lysis buffer (Promega, Madison, WI, USA). Luciferase activity was determined using an analytical luminescence luminometer according to the manufacturer's instructions. Luciferase activity was normalized for transfection efficiency using the corresponding $\beta$-galactosidase activity. All assays were performed at least in triplicate.

\section{Cell viability assay}

Cell viability was determined by PrestoBlue cell viability reagent (Invitrogen, Carlsbad, CA, USA). Cells were treated with sorafenib at the indicated concentration for $24 \mathrm{~h}$. The medium was removed and replaced with complete cell culture medium containing PrestoBlue $(\times 10)$ for $1 \mathrm{~h}$. After incubation with PrestoBlue, the medium was placed into 96-well plate for analysis. Absorbance values were determined at $570 \mathrm{~nm}$.

\section{Reverse transcriptase-PCR and real-time PCR}

Total RNAs from cells were prepared using Trizol (Invitrogen) according to the manufacturer's recommendation. The cDNA was synthesized from $0.5 \mu \mathrm{g}$ of total RNA with M-MLV reverse transcriptase (Promega) using oligo-dT at $37{ }^{\circ} \mathrm{C}$ for $1 \mathrm{~h}$. The one-twentieth aliquot of the cDNA was subjected to PCR amplification using gene-specific primers [Table 1]. The CDNAs were amplified by PCR and the PCR products were examined by electrophoresis on $1.2 \%$ agarose gel. Real-time PCR was performed with TOPreal $q$ PCR $\times 2$ PreMIX with SYBR green (Enzynomics, Daejeon, South Korea) and each of the primers using StepOne ${ }^{\text {tw }}$ Real-time PCR System (Applied Biosystems, Carlsbad, CA, USA). The comparative threshold cycle method ( $\Delta \Delta \mathrm{C}_{\mathrm{T}}$ method) was used to calculate the relative gene expression levels with human $\beta$-actin as an endogenous control gene.

\section{SDS-PAGE and Western blotting}

Cells were lysed with lysis buffer containing 150 $\mathrm{mmol} / \mathrm{L} \mathrm{NaCl}, 50 \mathrm{mmol} / \mathrm{L}$ Tris-Cl (pH 7.5), $1 \mathrm{mmol} / \mathrm{L}$ EDTA, $1 \%$ Nonidet P-40, 10\% glycerol and protease

Table 1: Primers used for PCR amplification

\begin{tabular}{llll}
\hline Speices & Gene & Type & Sequence (5'-3') \\
\hline HBV & HBx & Sense & ATGGCTGCTAGGCTGTGCTGC \\
& & Anti-sense & ACGGTGGTCTCCATGCGACG \\
& HBV core & Sense & ATGCAACTTTTTCACCTCTGC \\
& & Anti-sense & CTGAAGGAAAGAAGTCAGAAG \\
Human & FXR & Sense & GCCTGTAACAAAGAAGCCCC \\
& & Anti-sense & CAGTTACAAGCATTCAGCAAC \\
& \multirow{2}{*}{ B-actin } & Sense & GACTACCTCATGAAGATC \\
& & Anti-sense & GATCCACATCTGCTGGAA \\
\hline
\end{tabular}

PCR: polymerase chain reaction; HBV: hepatitis $B$ virus; FXR: farnesoid $X$ receptor 
inhibitor and $1 \mathrm{mmol} / \mathrm{L}$ PMSF. The protein concentration was determined by Bradford assay (Bio-Rad, Hercules, CA, USA). Equal amounts of protein were loaded and separated by sodium dodecyl sulfate-polyacrylamide gel electrophoresis, and the proteins were transferred on to a PVDF membrane (Millipore, Billerica, MA, USA). For Western blotting, the membranes were incubated with anti-actin (Sigma, Steinheim, Germany), anti-HBx (Chemicon, Danvers, MA, USA), anti-flag (Cell Signaling, Beverly, MA, USA), anti-SAPK/JNK (Cell Signaling), anti-p-SAPK/ JNK (Cell Signaling), anti-c-jun (Santa Cruz Biotechnology, Santa Cruz, CA, USA), anti-p-c-jun (Santa Cruz Biotechnology) or anti-FXR (Santa Cruz Biotechnology) antibodies in tris-buffered saline with Tween 20 (TBST) containing 1\% Tween 20 supplemented with 3\% nonfat dried milk. After washing with TBST, the blotted membranes were incubated with the peroxidase-conjugated secondary antibody (Santa Cruz Biotechnology). After washing TBST, the proteins were visualized by the ECL development reagent (Amersham Pharmacia Biotech, Piscataway, NJ, USA).

\section{Statistical analysis}

Statistical analyses were carried out by unpaired or paired $t$-test as appropriate. All data are reported as mean \pm standard deviation. $P<0.05$ were considered significant.

\section{RESULTS}

\section{Sorafenib suppresses HBV gene expression}

To investigate the anti-viral effect of sorafenib, we obtained promoters of genes contained in the HBV genome. As shown in Figure 1a, the HBV genome contains 4 promoters and 2 enhancers that regulate HBV replication. Of these, the most important one during HBV replication is the precore/core promoter, which has an effect on transcription of pregenomic RNA from cccDNA..$^{[5]}$ The $\times 1.3$ HBV-Cp-luc construct contains the core promoter [Figure 1a]. The promoter activities of the $\mathrm{HBV}$ core, $\mathrm{X}$, and preS1 genes were decreased by sorafenib in a dose-dependent manner [Figure 1b-d]. To investigate if the decrease of promoter activities by sorafenib was induced by cell death, a cell viability assay was performed. The results

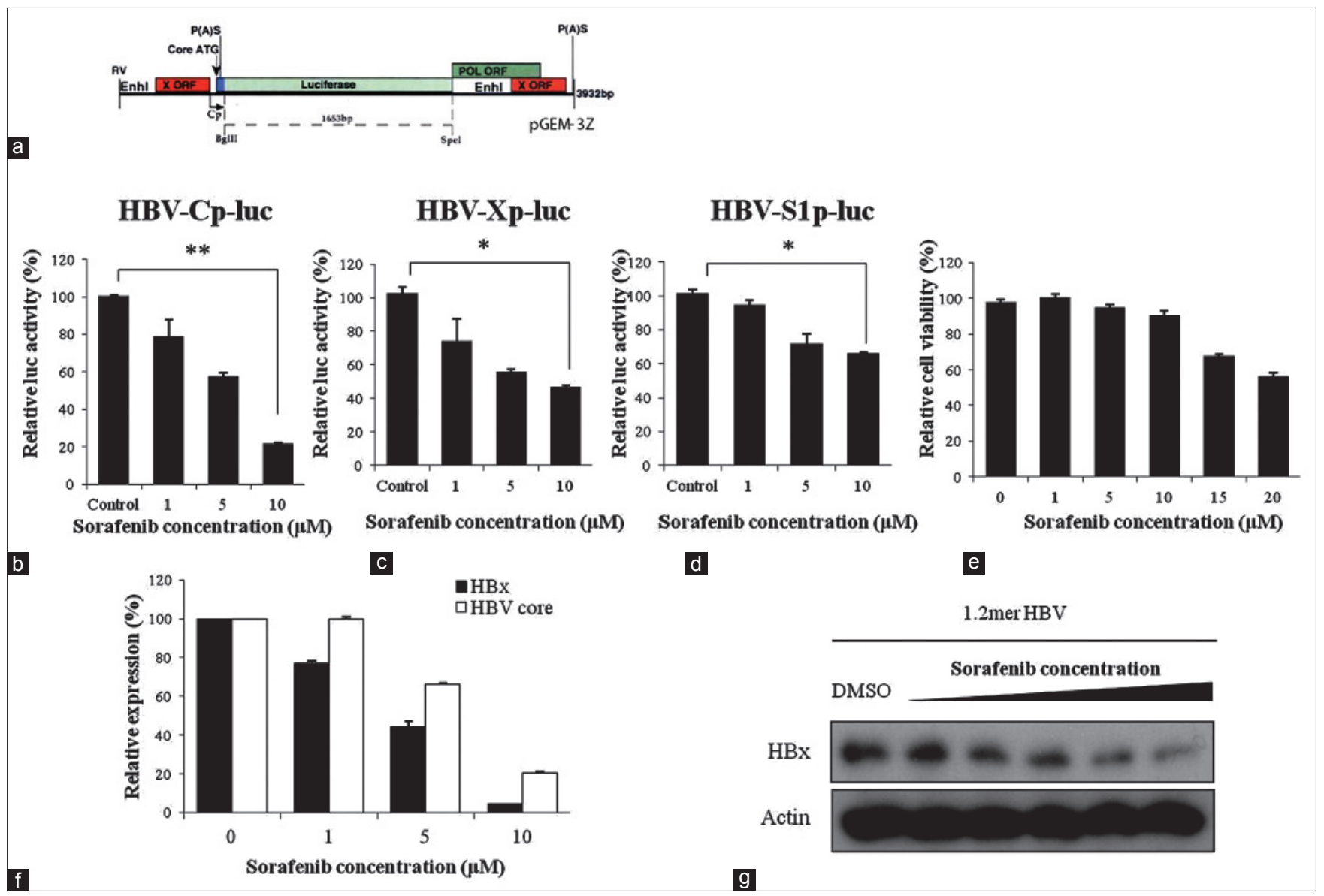

Figure 1: Sorafenib suppresses hepatitis B virus (HBV) gene expression. (a) Structure of the $\times 1.3 \mathrm{HBV}$-luc plasmid; (b-d) the effects of sorafenib on HBV promoter activity. HepG2 or Chang cells were transfected with $\times 1.3$ HBV-luc, pGL4-X or pGL4-preS1 constructs for $24 \mathrm{~h}$ and treated with sorafenib. The cell lysates were analyzed for luciferase activity. ${ }^{\star} P<0.05$, ${ }^{* *} P<0.01$, compared with the indicated cells; (e) cell viability assay; (f and g) the effect of sorafenib on HBV RNA or protein levels. HepG2 cells were transfected with 1.2 mer HBV construct for $24 \mathrm{~h}$ and treated with sorafenib. The indicated mRNA levels were detected by real-time polymerase chain reaction. Total proteins were prepared from the cells, and then HBx protein levels were detected by Western blotting 
showed that there was no cytotoxicity at $<10 \mu \mathrm{mol} / \mathrm{L}$ of sorafenib [Figure 1e]. In addition, mRNA and protein levels of HBx or core, HBV gene products, were decreased by sorafenib in a dose-dependent manner [Figure $1 \mathrm{f}$ and $\mathrm{g}$ ]. These results suggest that sorafenib may suppress HBV gene expression regardless of cell death.

\section{Sorafenib suppresses HBV gene expression through inhibition of the JNK pathway}

Sorafenib is a multikinase inhibitor and it blocks several kinase pathways. Of these, the JNK pathway has been reported to be inhibited by sorafenib ${ }^{[6]}$ and have a possibility to regulate HBV pathogenesis. ${ }^{[7]}$ Therefore, we investigated if the JNK pathway is blocked by sorafenib and HBV gene expression is suppressed by JNK inhibition. As expected, phosphorylation of JNK and HBV protein levels were decreased by sorafenib [Figure 2a]. This result showed an effect of sorafenib as a JNK pathway inhibitor. In addition, HBV core promoter activity was decreased with inhibition of the JNK pathway [Figure 2b]. HBV mRNA and protein levels were also decreased by inhibition of the JNK pathway [Figure 2c]. The protein levels of c-jun and phosphorylated c-jun were used as a target of the JNK pathway. Inversely, HBx protein levels were increased by JNK overexpression [Figure $2 \mathrm{~d}$ ] and HBV promoter activity and HBx expression induced by JNK1 were attenuated by sorafenib [Figure 2e and f]. These results suggest that sorafenib may suppress HBV gene expression through JNK pathway inhibition.

\section{Sorafenib suppresses FXR-induced HBV gene expression}

To identify the potential transcription factors increasing HBV gene expression and targeted by sorafenib, several hepatocyte-enriched transcription factors were assessed [Figure 3a]. Of these, FXR increased HBV promoter activity and FXR-induced HBV promoter activity was attenuated by sorafenib. FXR enhances synthesis of pregenomic RNA, FXR, and bile acids, the natural ligand of FXR related to the JNK pathway. ${ }^{[8]}$ Therefore, FXR was considered as a strong candidate targeted by sorafenib. FXR with CDCA, an endogenous FXR ligand, increased HBV core promoter activity [Figure $3 b$ ], HBV gene expression in mRNA, and protein levels without a change in FXR gene expression levels [Figure 3c]. To further investigate the effect of FXR on HBV gene expression,

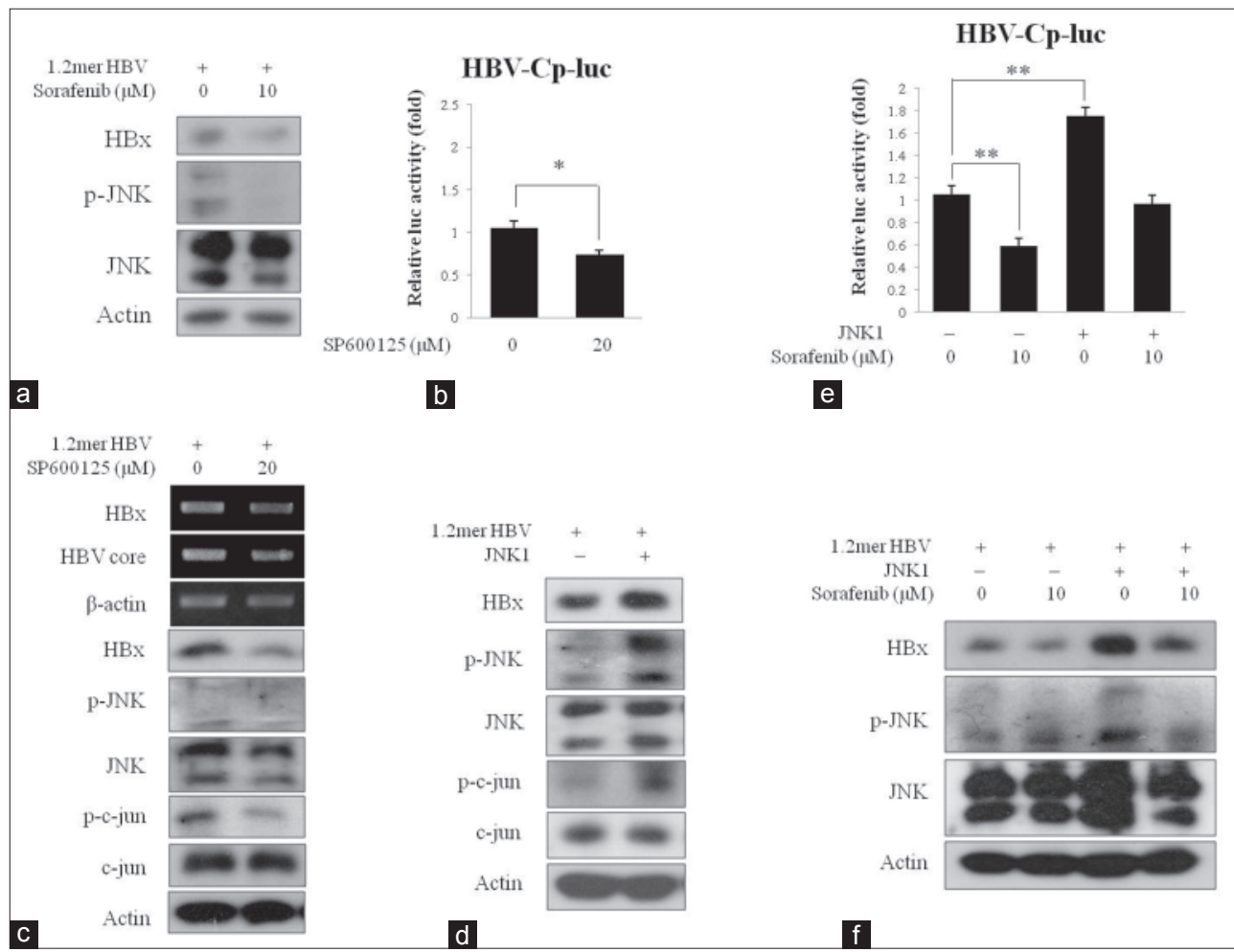

Figure 2: Sorafenib suppresses hepatitis B virus (HBV) gene expression through inhibition of the JNK pathway. (a) The effect of sorafenib on JNK phosphorylation and HBV protein levels. Chang cells were transfected with 1.2 mer HBV construct and treated with sorafenib. The indicated protein levels were detected by Western blotting; (b) the effect of JNK inhibitor on HBV core promoter activity. Chang cells were transfected with $\times 1.3$ HBV-luc construct and then maintained either control conditions or in the presence of SP600125. The cell lysates were analyzed for luciferase activity. ${ }^{*} P<0.05$ compared with the indicated cells; (c) the effect of JNK inhibitor on HBV gene expression. Chang cells were transfected with 1.2 mer HBV construct and treated with SP600125. The cells were analyzed by reverse transcriptase-polymerase chain reaction and Western blotting; (d) the effect of JNK on HBV gene expression. Chang cells were cotransfected with the indicated constructs. The indicated protein levels were detected by Western blotting; (e) the effect of sorafenib on JNK-induced HBV core promoter activity. Chang cells were cotransfected with the indicated constructs, and the cells were treated with sorafenib. The cell lysates were analyzed for luciferase activity. ${ }^{* *} P<0.01$ compared with the indicated cells; (f) the effect of sorafenib on JNK-induced HBV protein expression. Chang cells were cotransfected with the indicated constructs, and the cells were treated with sorafenib. The indicated protein levels were detected by Western blotting 
an antagonist of FXR, Z-GGS was used to inactivate FXR. HBx core promoter activity and HBV gene expression were decreased by FXR inactivation [Figure $3 \mathrm{~d}$ and e]. To address the mechanism by which sorafenib decreases FXR-induced HBV core promoter activity, we investigated FXR protein levels after sorafenib treatment. As a result, FXR protein levels were decreased by sorafenib [Figure 3f] and FXR-induced HBx protein expression was also decreased by sorafenib [Figure $3 g$ ]. These results suggest that sorafenib may suppress HBV gene expression induced by FXR.

\section{Sorafenib suppresses FXR-induced HBV gene expression} through inhibition of the JNK pathway

As shown above, sorafenib suppressed HBV gene expression,
FXR, and the JNK pathway. We tried to address the relationship of these molecules involved in suppressing HBV gene expression. First, FXR protein levels were decreased by JNK inhibition and increased by JNK overexpression, but mRNA levels of FXR were not affected [Figure 4a and b]. These results suggest that the JNK pathway affects FXR protein stability and activity, but not FXR gene expression. The promoter activities of HBV genes induced by FXR or activated FXR were attenuated by sorafenib [Figure 4c-e]. In addition, the expression levels of $\mathrm{HBx}$ and core genes were increased by FXR and FXR activated by CDCA, but the expression levels were attenuated by sorafenib [Figure $4 \mathrm{f}$ and $g$. These results suggest that sorafenib may suppress FXR-induced HBV gene expression through JNK pathway inhibition.

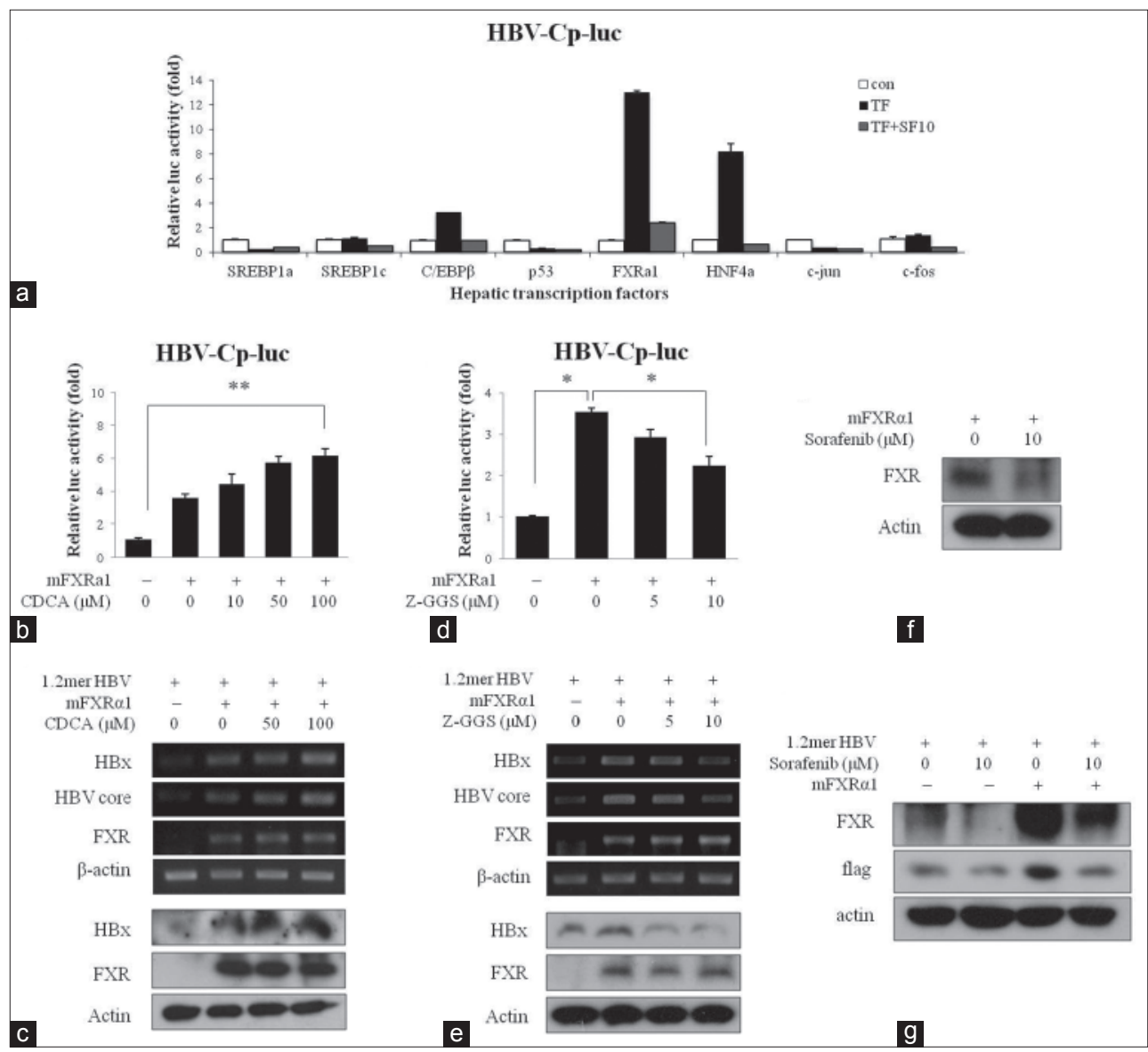

Figure 3: Sorafenib suppresses farnesoid X receptor (FXR)-induced hepatitis B virus (HBV) gene expression. (a) The effect of several hepatic transcription factors and sorafenib on HBV promoter activities. Chang cells were cotransfected with $\times 1.3 \mathrm{HBV}$-luc construct and the indicated transcription factors, and incubated in sorafenib. The cell lysates were analyzed for luciferase activity; (b) the effect of FXR $\alpha 1$ on HBV core promoter activity. Chang cells were transfected with the indicated constructs, and then maintained either control conditions or in the presence of chenodeoxycholic acid (CDCA). The cell lysates were analyzed for luciferase activity. ${ }^{* *} P<0.01$ compared with the indicated cells; (c) the effect of FXR on HBV mRNA and protein levels. Chang cells were transfected with the indicated constructs, and then maintained either control conditions or in the presence of CDCA. The indicated mRNA or protein levels were detected by reverse transcriptase-polymerase chain reaction (RT-PCR) or Western blotting; (d) the effect of FXR on HBV core promoter activity by the antagonist. Chang cells were transfected with the indicated constructs, and then maintained either control conditions or in the presence of Z-guggulsterone (Z-GGS). The cell lysates were analyzed for luciferase activity. ${ }^{*} P<0.05$ compared with the indicated cells; (e) the effect of FXR on HBV mRNA and protein levels by the antagonist. Chang cells were transfected with the indicated constructs, and then maintained either control conditions or in the presence of Z-GGS. The indicated mRNA or protein levels were detected by RT-PCR or Western blotting; ( $f$ ) the effect of sorafenib on FXR. Chang cells were transfected with pCMV-mFXR $\alpha 1$ construct, and then maintained either under control conditions or in the presence of sorafenib; $(\mathrm{g})$ the effect of sorafenib on FXR and HBV protein levels. Chang cells were transfected with the indicated constructs, and then maintained either control conditions or in the presence of sorafenib. The transfected cells were analyzed by Western blotting 


\section{DISCUSSION}

The antiviral effect of sorafenib on HBV gene expression indicates meaningful approaches to anti-HBV drugs. There are several available agents for the treatment of CHB. These drugs, including immunomodulatory agents and nucleotide/nucleoside analogs, have high efficacy against $\mathrm{CHB}$, but there are several limitations including side effects, tolerance, and drug resistance. ${ }^{|9|}$ These limitations have been considered as problems to overcome for at least 10 years. ${ }^{[10]}$ Currently, combined therapies of these drugs show improved efficacy and lower drug resistance in CHB. ${ }^{[11]}$ However, other drugs with new strategies for $\mathrm{HBV}$ replication have not been approved yet. The development of more effective drugs for the management of $\mathrm{CHB}$ has proven to be challenging. Here, sorafenib suppressed HBV gene expression by inhibiting the JNK signaling pathway. This new mechanism suggests another possible approach to inhibit HBV replication. Furthermore, sorafenib could be combined with other established anti-HBV agents to treat $\mathrm{CHB}$ because the combined therapy with other mechanisms is expected to show improved efficacy.

Sorafenib is a medicine for HCC and HCC is mainly caused by HBV infection. ${ }^{[12]}$ Therefore, we expect that sorafenib will help block the progress of hepatitis B and prevent the development of HCC. This might be a unique advantage as an antiviral drug because other currently available anti-HBV agents don't have oncomodulatory effects. In addition, targeting the host molecular signaling pathway is expected to have less drug-resistance compared to nucleotide/nucleoside analogs, which targets viral polymerase with high genetic variation. ${ }^{[9]}$ The mechanism of the effect of sorafenib on HBV gene expression should be elucidated with a variety of studies in vitro and in vivo. Especially, the effect of sorafenib on anti-HBV drug-resistant strains should be investigated through combined therapy.

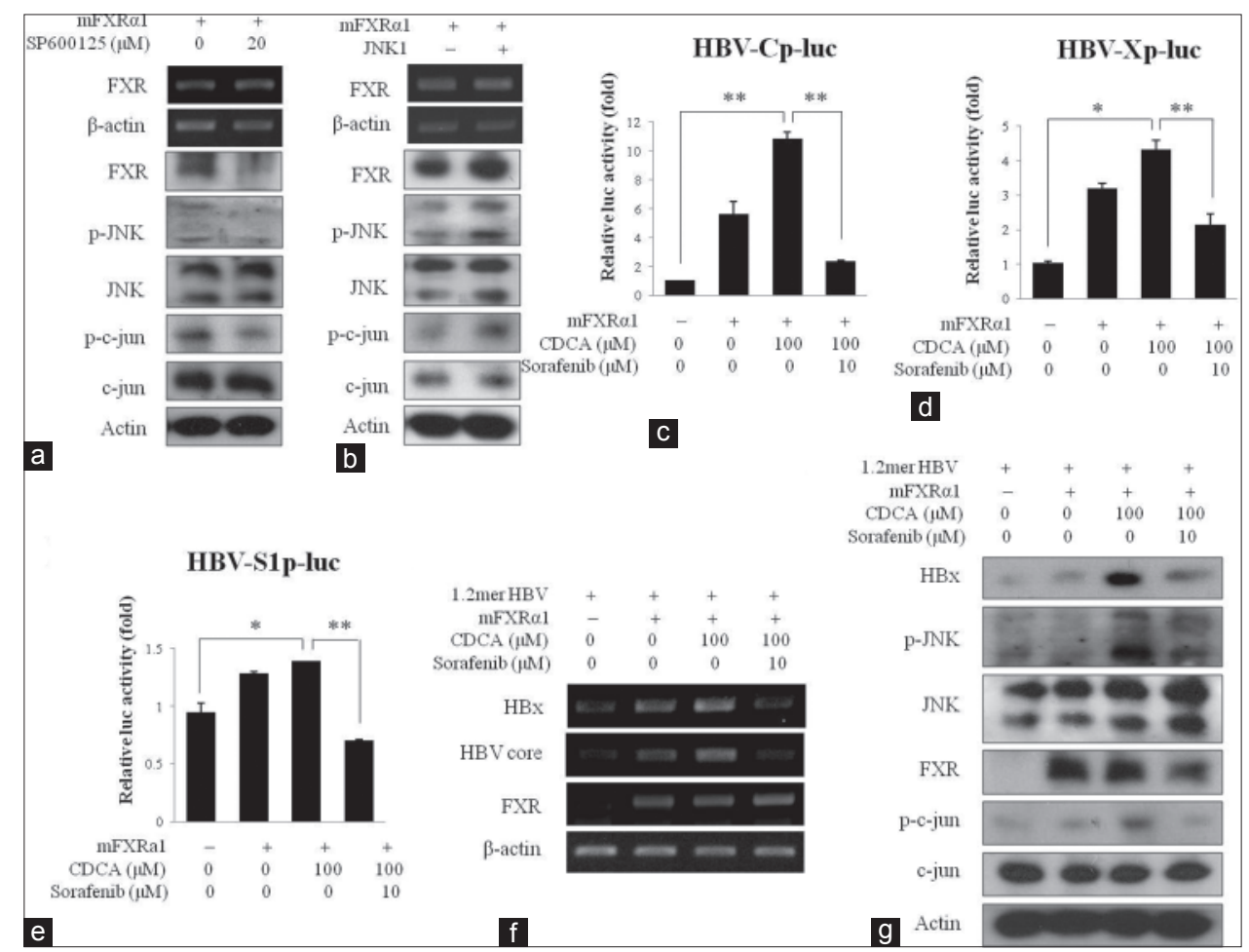

Figure 4: Sorafenib suppresses farnesoid X receptor (FXR)-induced hepatitis B virus (HBV) gene expression through inhibition of JNK pathway. (a) The effect of JNK inhibitor on FXR gene expression levels. Chang cells were transfected with pCMV-mFXR 1 construct and treated with SP600125. The indicated mRNA or protein levels were detected by reverse transcriptase-polymerase chain reaction (RT-PCR) or Western blotting; (b) the effect of JNK on FXR gene expression levels. Chang cells were transfected with pCMV-mFXR $\alpha 1$ construct and treated with SP600125. The indicated mRNA or protein levels were detected by RT-PCR or Western blotting; (c) the effect of sorafenib on FXR-induced HBV core promoter activity. Chang cells were transfected with the indicated constructs, and then maintained either control conditions or in the presence of chenodeoxycholic acid (CDCA) and sorafenib. The cell lysates were analyzed for luciferase activity. ${ }^{\star \star} P<0.01$ compared with the indicated cells; (d) the effect of sorafenib on FXR-induced HBV X promoter activity. Chang cells were transfected with the indicated constructs, and then maintained either control conditions or in the presence of CDCA and sorafenib. The cell lysates were analyzed for luciferase activity. ${ }^{\star} P<0.05$, ${ }^{* *} P<0.01$ compared with the indicated cells; (e) the effect of sorafenib on FXR-induced HBV preS1 promoter activity. Chang cells were transfected with the indicated constructs, and then maintained either control conditions or in the presence of CDCA and sorafenib. The cell lysates were analyzed for luciferase activity. ${ }^{*} P<0.05$, ${ }^{* *} P<0.01$ compared with the indicated cells; $(f)$ the effect of sorafenib on FXR-induced HBV mRNA levels. Chang cells were transfected with the indicated constructs as described on each lane, and the cells were treated with CDCA and sorafenib. The indicated mRNA levels were detected by RT-PCR; ( $g$ ) the effect of sorafenib on FXR-induced HBV protein levels. Chang cells were transfected with the indicated constructs as described on each lane, and the cells were treated with CDCA and sorafenib. The indicated protein levels were detected by Western blotting 
Bile acid activates the JNK pathway and FXR, which suppresses cholesterol-7 $\alpha$-hydroxylase (CYP7A1), independently. ${ }^{[8]}$ Bile acids are known to inhibit CYP7A1 gene transcription via direct activation of the JNK pathway, and FXR activates the JNK pathway to suppress CYP7A1 in hepatocytes via inducing intestinal fibroblast growth factor 15/19. In this study, JNK activation affected FXR transcriptional activity and FXR protein levels but not FXR mRNA levels, suggesting that the JNK pathway may regulate FXR transcriptional activity and protein stability.

Sorafenib is a well-known Raf kinase inhibitor, but it also inhibits other kinases. ${ }^{[13]}$ The JNK pathway is one of the signaling pathways inhibited by sorafenib. However, sorafenib has been reported to activate the JNK pathway to induce apoptosis. ${ }^{[14]}$ It seems to be in conflict with each other, but the effect of sorafenib in a nontoxic concentration was confirmed in this study. Therefore, it may be important to use a proper dose of sorafenib to treat CHB. In this study, the proper dose of sorafenib was $<10 \mu \mathrm{mol} / \mathrm{L}$. In the other case, the proper dose of sorafenib that inhibits human cytomegalovirus replication and hepatitis $C$ virus was below 2.5 and $15 \mu \mathrm{mol} / \mathrm{L}$ in vitro, respectively. ${ }^{[15,16]}$

In conclusion, these findings suggest that sorafenib, the multi-kinase inhibitor, has an anti-viral effect on HBV gene expression. Further research about the efficacy of targeting the molecular signaling pathway of HBV replication should be evaluated for the treatment of $\mathrm{CHB}$ patients.

\section{ACKNOWLEDGMENTS}

This work was supported by a 2-Year Research Grant of Pusan National University.

\section{REFERENCES}

1. Kao JH, Chen DS. Global control of hepatitis B virus infection. Lancet Infect Dis 2002;2:395-403.

2. Liang TJ.Hepatitis B: the virus and disease. Hepatology 2009;49:S13-21.

3. Liu L, Cao Y, Chen C, Zhang X, McNabola A, Wilkie D, Wilhelm S, Lynch M, Carter C. Sorafenib blocks the RAF/MEK/ERK pathway, inhibits tumor angiogenesis, and induces tumor cell apoptosis in hepatocellular carcinoma model PLC/PRF/5. Cancer Res
2006; $66: 11851-8$

4. Borgia G, Gentile I. Treating chronic hepatitis B: today and tomorrow. Curr Med Chem 2006;13:2839-55.

5. Gilbert S, Galarneau L, Lamontagne A, Roy S, Bélanger L. The hepatitis $\mathrm{B}$ virus core promoter is strongly activated by the liver nuclear receptor fetoprotein transcription factor or by ectopically expressed steroidogenic factor 1. J Virol 2000;74:5032-9.

6. Nakamura K, Hatano E, Narita M, Miyagawa-Hayashino A, Koyama Y, Nagata H, Iwaisako K, Taura K, Uemoto S. Sorafenib attenuates monocrotaline-induced sinusoidal obstruction syndrome in rats through suppression of JNK and MMP-9. J Hepatol 2012;57:1037-43.

7. Srisuttee R, Koh SS, Malilas W, Moon J, Cho IR, Jhun BH, Horio Y, Chung YH. SIRT1 sensitizes hepatocellular carcinoma cells expressing hepatitis B virus $\mathrm{X}$ protein to oxidative stress-induced apoptosis. Biochem Biophys Res Commun 2012;429:45-50.

8. Li T, Jahan A, Chiang JY. Bile acids and cytokines inhibit the human cholesterol 7 alpha-hydroxylase gene via the $\mathrm{JNK} / \mathrm{c}$-jun pathway in human liver cells. Hepatology 2006;43:1202-10.

9. Fung J, Lai CL, Seto WK, Yuen MF. Nucleoside/nucleotide analogues in the treatment of chronic hepatitis B. J Antimicrob Chemother 2011;66:2715-25.

10. Pramoolsinsup C. Management of viral hepatitis B. J Gastroenterol Hepatol 2002;17 Suppl: S125-45.

11. Terrault NA. Benefits and risks of combination therapy for hepatitis B. Hepatology 2009;49:S122-8.

12. Xie B, Wang DH, Spechler SJ. Sorafenib for treatment of hepatocellular carcinoma: a systematic review. Dig Dis Sci 2012;57:1122-9.

13. Karaman MW, Herrgard S, Treiber DK, Gallant P, Atteridge CE, Campbell BT, Chan KW, Ciceri P, Davis MI, Edeen PT, Faraoni R, Floyd M, Hunt JP, Lockhart DJ, Milanov ZV, Morrison MJ, Pallares G, Patel HK, Pritchard S, Wodicka LM, Zarrinkar PP. A quantitative analysis of kinase inhibitor selectivity. Nat Biotechnol 2008;26:127-32.

14. Rahmani M, Davis EM, Crabtree TR, Habibi JR, Nguyen TK, Dent P, Grant S. The kinase inhibitor sorafenib induces cell death through a process involving induction of endoplasmic reticulum stress. Mol Cell Biol 2007;27:5499-513.

15. Michaelis M, Paulus C, Löschmann N, Dauth S, Stange E, Doerr HW, Nevels M, Cinatl J Jr. The multi-targeted kinase inhibitor sorafenib inhibits human cytomegalovirus replication. Cell Mol Life Sci 2011;68:1079-90.

16. Himmelsbach K, Sauter D, Baumert TF, Ludwig L, Blum HE, Hildt E. New aspects of an anti-tumour drug: sorafenib efficiently inhibits HCV replication. Gut 2009;58:1644-53.

How to cite this article: Cho HK, Kim JR, Kim SY, Kyaw YY, Win AA Cheong $\mathrm{JH}$. Sorafenib suppresses hepatitis $B$ virus gene expression via inhibiting JNK pathway. Hepatoma Res 2015;1:97-103.

Source of Support: This work was supported by a 2-Year Research Grant of Pusan National University. Conflict of Interest: None declared. 